\title{
Riskante Verläufe im Wochenbett
}

Christiane Borchard, Hebamme, Familienhebamme, Dipl. Pflegewirtin (FH), Münster

\section{Dramatische Verläufe im Wochenbett sind glücklicherweise selten. Die Schattenseite davon ist, dass Hebammen keine Routine mit ihnen entwickeln können. Umso mehr kommt einem durchdachten Risikomanagement eine bedeutende Rolle für die Wochenbettbetreuung zu.}

Für jedes Risikomanagement - auch für das im Wochenbett - ist ein gut funktionierendes Team wichtig. Die meisten Hebammen stehen beim Hausbesuch jedoch alleine da - auch bei Komplikationen. Das Team, auf das sie zurückgreifen könnten, besteht aus Mitarbeitern und Mitarbeiterinnen in den Krankenhäusern sowie niedergelassenen Ärzten.

\section{Umgang mit Risiken im Berufsalltag}

Hebammen suchen zunächst systematisch nach Risiken und bauen Sicherheitsbarrieren auf, damit sich die Risiken in der Praxis nicht ausbreiten und sich ungebremst potenzieren können. Ziel ist es, diese Sicherheitsbarrieren möglichst dicht einzurichten. Der britische Psychologe James Reason entwickelte das Schweizer-Käse-Modell [1]. Es veranschaulicht, dass jede Barriere Sicherheitslücken haben kann, löchrig sein kann wie die Scheiben eines Schweizer Käses: Wenn die Käsescheiben unglücklich übereinander liegen - also die Sicherheitsbarrieren lückenhaft sind - kann eine negative Ereigniskette ungehindert zu einem unerwünschten Ereignis führen.

Die Sicherheitsbarrieren der Hebammenpraxis bestehen z.B. aus:

- aktuellem Fachwissen

- klaren Kommunikationsstrukturen

- guter Praxisorganisation

- geregelter Zusammenarbeit mit Kolleginnen und anderen Berufsgruppen

\section{Qualitätsmanagement}

Das Risikomanagement in der Hebammenpraxis ist im Qualitätsmanagement-System (QM-System) verankert und wird im QM-Ordner dokumentiert. Hier besteht die Möglichkeit, Verbesserungen u.a. in den fachlichen, organisatorischen und strukturellen Bereichen zu planen, zu prüfen und in die alltägliche Praxis einzubauen.
Die individuelle Verlaufsdokumentation der betreuten Frauen ist wichtig, um medizinische Risiken und Abweichungen wahrzunehmen. Dazu gehört das Erheben der Anamnese: Frauen nach Vorerkrankungen zu fragen, bedeutet, medizinische Risiken wahrzunehmen. Im weiteren Verlauf zählt das Beobachten, Beschreiben und Bewerten von Abweichungen dazu.

Das Dokumentationssystem der Hebammen ist in den vergangenen Jahren deutlich umfangreicher geworden. Viele sind dazu übergegangen, spezielle Dokumentationsbögen zu benutzen, z.B. den Ikterusbogen, in dem Hautkolorit, Laborwerte und Parameter für die kindliche Vitalität standardisiert erfasst werden. Farbfelder im Formular helfen bei der Risikobewertung: Die Intensität des Farbtons zeigt das zunehmende Gefährdungspotenzial.

Hebammen reflektieren bei jedem Hausbesuch individuelle Risiken:

- Handelt es sich noch um Routine?

- Gibt es leichte Abweichungen, besteht ein Risiko?

- Gibt es gravierende Abweichungen, braucht die Frau evtl. einen Facharzt oder muss sie eine Klinik aufsuchen?

Leider ist es in der Praxis bei einigen Verläufen nicht so leicht, die klare Trennschärfe zwischen Physiologie und Pathologie zu ziehen. Deshalb müssen Hebammen sich absichern, indem sie z.B. bei einem Kind mit Verdacht auf Ikterus einen zweiten Wochenbettbesuch am gleichen Tag machen oder das Kind gleich wieder zur Verlaufskontrolle schicken. Bei Unsicherheiten kann auch ein Blick in die entsprechende Leitlinie Orientierung geben.

\section{Leitlinien}

Es gibt in Deutschland leider keine Leitlinie, die die lange und komplexe Wochenbettbetreuung komplett beleuchtet. Dafür gibt es einzelne Leitlinien, z.B. zur Therapie entzündlicher Brusterkrankungen in der Stillzeit [3], zur Hyperbilirubinämie [4] oder zu peripartalen Blutungen [5]. Diese sind allerdings überwiegend 
klinisch ausgerichtet und müssen für die Arbeit im Wochenbett von den Hebammen angepasst werden. Daher muss die Hebamme sich das Wissen oder die Orientierungshilfen für das Wochenbett aus verschiedenen Quellen aneignen.

In Großbritannien hat hingegen das National Institute for Health and Care Excellence (NICE) eine interdisziplinäre Leitlinie für das Wochenbett erstellt [6]. Diese enthält einen Abschnitt zum Notfallmanagement, der zusammenfasst, worüber Frauen im frühen Wochenbett aufgeklärt werden müssen.

\section{AUFKLÄRUNG ÜBER LEBENSBEDROHLICHE} EREIGNISSE IM WOCHENBETT (UK)

- Alle Wöchnerinnen erhalten innerhalb der ersten 24 Stunden postpartal eine Aufklärung über Anzeichen und Symptome lebensbedrohlicher Ereignisse

- Die Aufklärung beinhaltet präzise, evidenzbasierte, mündliche und schriftliche Informationen

Quelle: Leitlinie CG37 Postnatal Care, NICE 2013 [6]

Die Empfehlungen der britischen Leitlinie basieren u.a. auf den Ergebnissen der dreijährlich durchgeführten Müttersterblichkeitsberichte. Sie erlauben Rückschlüsse auf die Todesursachen der Mütter und können zur Prävention genutzt werden.

In Deutschland hingegen fehlen aussagekräftige Zahlen darüber, wie viele Frauen im Kontext Schwangerschaft, Geburt und Wochenbett sterben und woran sie gestorben sind. Aufgrund der fehlenden Fallanalysen ist es schwierig, Handlungsempfehlungen zu formulieren.

\section{Risikoaufklärung im Wochenbett}

\section{Situation in Großbritannien}

Konkret klären die Briten im Wochenbett u.a. gezielt auf über:

- potenziell lebensbedrohliche Risiken im Wochenbett, z.B. schwere Blutungen im Wochenbett, Sepsis,

Thrombosen, hypertensive Erkrankungen und Allergien

- akute Erkrankungen des Neugeborenen: Infekt/Sepsis, pathologischer Ikterus

- psychische Störungen mit akuter Eigen- oder Fremdgefährdung (wird im Verlauf von Schwangerschaft oder im Krankenhaus thematisiert, sofern sich in der Anamnese Auffälligkeiten ergeben)

Das sind keine leichten Themen für Gespräche mit Eltern und könnten bei diesen weitere Ängste und Unsicherheiten hervorrufen. Doch diese Themen haben eine große Relevanz - und Hebammen haben das Potenzial, damit feinfühlig und sensibel umzugehen. Das gelingt vor allem dann, wenn sie selbst diese Themen als relevant identifiziert haben.

\section{Situation in Deutschland}

In Deutschland verpflichtet das Patientenrechtegesetz in § 630a ff BGB Hebammen zur Aufklärung. Auch Schwangere und Wöchnerinnen haben ein Recht auf Aufklärung. Es gibt jedoch keine standardisierten Vorgaben dafür, wer wann worüber aufklärt: Die Hebamme beim Vorgespräch/bei der Anamnese? Die entlassende Klinik beim Abschlussgespräch? Die Hebamme beim ersten Hausbesuch im Wochenbett? Die Hebamme, wenn Abweichungen auftreten?

Das Problem: Im Wochenbett beschäftigen die Familien so viele anderen Themen, dass sich die Frage stellt, wie viele Informationen die Frau in diesem Moment überhaupt verarbeiten kann.

\section{Merke \\ Sinnvoll ist es, in das Gespräch mit der Frau einzu- steigen mit der Frage: „Was wissen Sie denn bereits über ... ? “ Die Hebamme kann dann fehlende Infor- mationen ergänzen oder falsche richtig stellen. Auf diese Weise bekommt sie ein Gespür dafür, ob die Frau zu diesem Zeitpunkt am Thema Risiken inte- ressiert ist.}

\section{Unterschätzte Risiken}

Unter den häufigsten Todesursachen britischer Mütter finden sich neben Wochenbetterkrankungen auch schwere Grunderkrankungen, die im Wochenbett zum Tod führen können. Die häufigsten Todesursachen sind: Krebs, Epilepsie, Sepsis, Schlaganfälle, Herzerkrankungen oder Thrombosen. Sie sind für gut drei Viertel der Todesfälle bis zum 42. Tag nach der Geburt verantwortlich. Ein knappes Viertel der Todesfälle $(23,5 \%)$ stehen in Zusammenhang mit psychischen Erkrankungen [7].

\section{Sepsis}

Während Kindbettfieber noch vor 100 Jahren als tödliche Erkrankung gefürchtet war, sterben heute kaum noch Frauen an einer Sepsis. Doch das Krankheitsbild hat nichts an Schrecken verloren. Denn die den für die schlimmsten Verläufe verantwortlichen Erreger Streptokokken A gibt es noch immer. Das Risiko besteht darin, dass Hebammen und Ärzte diese Erkrankung so selten sehen, dass sie die ersten Anzeichen diesem Krankheitsbild oft nicht zuordnen können.

Zu den ersten Anzeichen einer Sepsis gehört u.a. ein sich rasch verschlechternder Allgemeinzustand der Frau, der u.a auch durch diffuse Beschwerden im Bauchraum, Blähungen und Durchfall begleitet werden kann. Das kann durchaus auch mit dem Beginn einer 
Norovirusinfektion verwechselt werden. So kommt es zu einem verzögerten Behandlungsbeginn. Das kann fatale Folgen haben.

Auch bei einer Scharlach-Erkrankung in der Familie ist Vorsicht geboten. Es kann zu einer Übertragung von Streptokokken A kommen. Besucht die Hebamme eine fiebrige Wöchnerin, vermutet sie eher einen grippalen Infekt, wenn sie die Scharlacherkrankung des Kindes nicht im Blick hat. Das kann ebenfalls fatale Folgen haben, denn Streptokokken A führen innerhalb weniger Stunden zu einem ausgeprägten Krankheitsbild. Wenn die Hebamme dann mit der Frau einen zweiten Besuch für den Nachmittag vereinbart, kann es unter Umständen schon zu spät sein.

Bei einer fiebrigen Erkrankung der Frau im Wochenbett sollte eine Sepsis als mögliche Ursache mitbedacht werden. Die Ursache von Fieber muss immer abgeklärt werden.

\section{Thrombosen}

Frauen mit einem Body-Mass-Index (BMI) über 30 (oder gar über 40) haben ein erhöhtes Thrombose-Risiko insbesondere in Kombination mit einer Sectio. Denn eine Sectio erhöht das Thromboserisiko und in Kombination mit Übergewicht steigt es noch mehr. Für diese Frauen reicht möglicherweise eine Therapie mit niedrigmolekularem Heparin über 5-7 Tage nicht aus. Das Thromboserisiko ist im Wochenbett deutlich höher als in der Schwangerschaft - und zwar bis zu 12 Wochen postpartum [8].

Das Risiko verringert sich in diesem Zeitraum auch nicht, denn es ist unwahrscheinlich, dass die Frau in so kurzer Zeit 10 bis 15 Kilo abnimmt. Nach der Geburt wird eine erneute Bewertung des Risikos für eine Thrombose im weiteren Verlauf des Wochenbettes durchgeführt und bei Bedarf vom Facharzt eine 68 Wochen lange Heparintherapie angeordnet.

\section{Psychische Probleme}

Erschreckend ist, dass 23\% der Todesfälle auf mentale oder psychische Probleme zurückzuführen sind diese Zahl beinhaltet auch Tode z.B. infolge von Abusus.

Da psychische Probleme eine andere Dynamik aufweisen als andere Krankheiten, haben die Briten den Zeitraum für diese Fälle bis zum Ende des 1. Lebensjahres ausgedehnt. Dabei haben sie festgestellt, dass ein Viertel der Frauen aufgrund psychischer Krankheiten stirbt. Zwar sind die absoluten Zahlen gering, aber psychische Erkrankungen sind die häufigste Todesursache von Müttern: Jeder 7. Todesfall ist ein Selbstmord [7]. 
Bereits 2011 veröffentlichten die Briten, dass Sepsis und Suizid zu den häufigsten Todesursachen gehören [9].

Obwohl die postpartale Depression mit einer Häufigkeit von 10 bis 15\% zu den häufigsten Komplikationen im Wochenbett zählt, haben Hebammen sie in der Wochenbettbetreuung seltener im Blick. Zum Vergleich: Eine Mastitis bekommen nur 1-10\% der Wöchnerinnen [10], aber Hebammen klären in der Praxis häufiger darüber auf. Tritt in der Praxis das Ereignis Milchstau auf, vielleicht sogar mehrfach, fallen vermutlich schnell Begriffe wie Brustentzündung oder Mastitis. Betreut sie hingegen eine Frau, die über längere Zeit erschöpft ist, unter Schlafmangel leidet und sich schwer konzentrieren kann, ist fraglich, ob die Hebamme dann ebenso schnell auf eine mögliche Depression oder depressive Verstimmung zu sprechen kommt. Die meisten Hebammen würden eher darauf hinwirken, dass die Frau mehr Schlaf bekommt. Natürlich ist das zunächst segensreich, aber eine Depression lässt sich durch ausreichend Schlaf nur zum Teil mildern.

Immerhin erkranken in Deutschland jedes Jahr 100.000 Mütter an Depressionen [11]. Bei rund 700.000 Geburten pro Jahr bedeutet dies, dass jede 7. Frau von einer leichten, mittleren oder schweren Depression betroffen ist.

Die Mitglieder der Marcé-Gesellschaft, einer Fachgesellschaft für peripartale psychische Erkrankungen, beklagt: Peripartale psychische Störungen bleiben zum größten Teil unerkannt, sie werden unterschätzt und sie bleiben unbehandelt [12].

Unerkannt heißt: Lediglich 20 \% der betroffenen Frauen erhalten eine Verdachtsdiagnose oder eine Diagnose - gestellt von Gesundheitsfachberufen, Psychologen oder Hebammen (wobei Hebammen nur einen Verdacht äußern). Von den übrigen $80 \%$ versucht etwa ein Drittel, selber Hilfe zu finden. Diese Frauen erleben oft, dass sie nicht ernst genommen werden. [13]

Zum Thema postpartale psychische Erkrankungen gehört auch ein Hinweis auf den aktuellen Strukturmangel bei der Versorgung betroffener Frauen. Selbst wenn eine Hebamme sieht, dass eine Frau eine Depression entwickelt oder entwickelt hat, kann sie ihr oft nicht helfen: Denn für Therapieplätze im ambulanten oder im stationären Bereich gibt es heute lange Wartezeiten und die Unterbringung von Mutter und Kind in einer spezifischen Therapie ist kaum möglich. Dazu gibt es leider kaum Zahlen. Ein Experte aus NRW erzählte mir von Therapieplätzen für $20 \%$ der Betroffenen, d.h. einer Unterdeckung von 80\%. Diese Unterversorgung löst nicht nur bei den Frauen und ihren Familien Hilflosigkeit, Unsicherheit und Ohnmacht aus, sondern bei allen, die die Frauen betreuen.
EMPFEHLUNG FÜR DIE AUFKLÄRUNG ÜBER LEBENSBEDROHLICHE RISIKEN IM WOCHENBETT

Wenn Hebammen über Risiken im Wochenbett aufklären, ist es sinnvoll, sich am Beispiel Großbritannien zu orientieren und die lebensbedrohlichen Risiken mit in den Blick zu nehmen:

- Alle Frauen erhalten eine generelle Risikoaufklärung über die Anzeichen lebensbedrohlicher Situationen im Wochenbett. Es würde sich anbieten, den Eltern ein Infoblatt mit den wichtigsten Symptomen und Adressen der Krankenhäuser in der Region zu geben.

- Die Aufklärung könnte beim Vorgespräch, bei der Anamnese, dem ersten Wochenbettbesuch oder beim Auftreten erster Abweichungen stattfinden.

- Die Frau bekommt sowohl schriftliche als auch mündliche Informationen plus relevante Ansprechpartner mit Kontaktdaten der regionalen Krankenhäuser.

- Weitere Beratung/Begleitung erfolgt mit Blick auf die individuellen anamnestischen Risiken bzw. auf den Wochenbettverlauf.

Das ist eine sehr umfassende Anforderung an Hebammen. Sicher ist es sinnvoll, wenn sich Hebammen zunächst untereinander austauschen und dann in den interdisziplinären Diskurs gehen. Wünschenswert ist ein regional einheitliches Vorgehen, damit die Frauen von allen Versorgern die gleichen Informationen bekommen.

\section{Kommunikationskanäle}

Die Flut heutiger Kommunikationsmedien führt nicht unbedingt zu klarerer Kommunikation. Es gehört zum Alltag einer freiberuflichen Hebamme, morgens eine Reihe von WhatsApp-Nachrichten zu lesen und zu sortieren, die Wöchnerinnen in der Nacht geschrieben haben. Standards, wie damit umzugehen ist, gibt es nicht. So muss die Hebamme individuell entscheiden, was sie tut, wenn sie Abweichungen feststellt. Ob sie der Frau rät, einen Facharzt aufzusuchen oder ob sie ihn selbst anruft bzw. ihm eine E-Mail schickt.

Der Alltag sieht meist so aus: Die Hebamme erklärt der Frau, dass und warum sie zum Arzt gehen soll. Der Arzt legt indessen der Frau seine eigene Einstellung zum Problem dar. Diese kommt damit wieder zur Hebamme. Die Kommunikation findet also über die Frau statt - und birgt das Risiko, dass Informationen verloren gehen oder falsch weitergegeben werden wie beim Stille-Post-Spiel. Das trägt nicht zur Sicherheit bei.

Der Bayerische Hebammen Landesverband (BHLV) hat mit dem Berufsverband der Kinder- und Jugendärzte (bvkj) und PaedNetzBayern einen sehr guten Mitteilungsbogen 
erarbeitet. Das Formular mit dem Titel „Informationen Ihrer Hebamme für den Kinder- und Jugendarzt“ können Hebammen ausfüllen und der Frau zum Arztbesuch - auch bei ihrer Gynäkologin - mitgeben. Es steht auf der Webseite des BHLV zum Download bereit: www.bhlv.de. Darin können erkannte Gesundheitsstörungen/Probleme angekreuzt, durchgeführte Maßnahmen eingetragen und Empfehlungen/Wünsche an den Arzt mitgeteilt werden. Vom Arzt sind anschließend Diagnose und Therapiewahl einzutragen sowie der Hinweis, ob eine erweiterte Abklärung bzw. ein weiterer Kontrolltermin bei Arzt oder Hebamme notwendig ist. Zudem tragen Hebamme und Arztpraxis ihre Kontaktdaten und den bevorzugten Kommunikationsweg ein (Telefon, Fax, Mail). In Bayern wird bereits erfolgreich mit dem Mitteilungsbogen gearbeitet.

Das ist ein gutes Praxisbeispiel, mit dem die Kommunikation deutlich optimiert werden kann. Ein erster Austausch mit Kinderärzten ergab, dass sie damit gut arbeiten können, denn sie wünschen sich Informationen über die Verläufe im Wochenbett, die über Gedeihstörungen, Gewichtsverlauf oder Stillen hinausgehen. Oft hilft ihnen die Einschätzung der Hebamme sehr.

\section{Risiko: Mastitis}

Eine weit verbreitete Komplikation im Wochenbett ist der Milchstau - der Übergang zur Mastitis. Die Leitlinie zur Therapie entzündlicher Brusterkrankungen in der Stillzeit der DGGG [3] kann eine Orientierungshilfe bieten ( $\triangleright$ Tab.1).

Einschränkend ist festzustellen, dass sich die Übergänge in der Theorie klarer trennen lassen als in der Praxis, wo die Übergänge häufig fließend sind. Dazu muss man sich darüber im Klaren sein, dass eine Leitlinie die bunte Vielfalt der täglichen Praxis nicht abbilden kann. Im individuellen Einzelfall kann z.B. eine Mastitis bereits vorliegen, auch wenn bei der Stillenden noch keine deutlich erhöhte Körpertemperatur über $38,4^{\circ} \mathrm{C}$ vorliegt.

Häufig schicken Frauen den Hebammen Fotos, die ein Problem zeigen, etwa eine Hautrötung auf der Brust, damit die Hebamme einen Rat erteilen kann. Doch ist es sinnvoll, anhand eines Fotos eine Diagnose zu stellen? Wenn Hebammen das machen, dann handelt es sich um Telemedizin. Hebammen sollten sich sehr genau überlegen, ob sie das machen wollen, denn jeder Bildschirm zeigt beispielsweise die Rötung etwas anders an. So darf bezweifelt werden, ob sich anhand eines Bildes wirklich sagen lässt, dass es sich um einen Milchstau oder eine Mastitis handelt. Für die genaue Diagnostik muss die Hebamme erst mit der Frau telefonieren, dann einen Hausbesuch machen.

\section{BEISPIEL}

\section{Rasanter Verlauf bei Mastitis}

Eine junge Mutter wird wenige Tage nach der Geburt mit deutlich ausgeprägten Rhagaden beidseits aus der Klinik entlassen. Die Frau litt bereits im frühen Wochenbett an Vasospasmus oder Raynauld-Syndrom. Aus diesem Grund dauerte es auch rund 10 Tage, bis die Brustwarzen wieder verheilt waren, die Eintrittspforte für Keime geschlossen war.

Am 12. Wochenbetttag macht die Hebamme morgens einen Hausbesuch und stellt keine Auffälligkeiten fest. Zwei bis drei Stunden später bekommt die Wöchnerin plötzlich $38,4^{\circ} \mathrm{C}$ Fieber, das rasch steigt, begleitet von heftigem Schüttelfrost. Die Frau sucht zeitnah die nächste Klinik auf.

Die Hebamme ist erschrocken über den ungewöhnlich rasanten Verlauf und vermutet, dass der vorliegende ausgeprägte Vaso-Spasmus (RaynauldSyndrom) zu diesem Ereignis geführt hat.

Tab. 1

\begin{tabular}{|c|c|}
\hline Milchstau & Mastitis \\
\hline lokale Schmerzen & lokale Schmerzen in der Brust \\
\hline lokale Verhärtung (Knoten) & $\begin{array}{l}\text { örtlich begrenzte, gerötete, überwärmte und geschwollene Bereiche der } \\
\text { Brust }\end{array}$ \\
\hline Keine oder leichte Überwärmung der betroffenen Stellen & systemische Reaktionen wie Unwohlsein und Fieber $\left(>38,4^{\circ} \mathrm{C}\right)$ \\
\hline kein Fieber $\left(<38,4^{\circ} \mathrm{C}\right)$ & lokale Symptome meist unilateral, in selterneren Fällen bilateral \\
\hline \multicolumn{2}{|l|}{ gutes Allgemeinbefinden } \\
\hline \multicolumn{2}{|l|}{ unilateral } \\
\hline zum Teil ein kleines weißes Bläschen auf der Mamille & \\
\hline
\end{tabular}


- Tab. 2 Geschätzte Inzidenz für schwere Hyperbilirubinämien [4].

\begin{tabular}{|l|l|l|}
\hline Betroffene Kinder & Wert/Krankheitsbild & \\
\hline 1 von 1000 & $>25 \mathrm{mg} / \mathrm{dl}$ & $\begin{array}{l}\text { Bei intensiver und zeitnaher Fototherapie, ggf. Austauschtransfusion(<5\%) entste- } \\
\text { hen in der Regel keine neurologischen Schäden }\end{array}$ \\
\hline 1 von 10.000 & $>30 \mathrm{mg} / \mathrm{dl}$ & $\begin{array}{l}\text { Bei intensiver und zeitnaher Fototherapie, ggf. Austauschtransfusion(<5\%) entste- } \\
\text { hen in der Regel keine neurologischen Schäden }\end{array}$ \\
\hline 1 von 100.000 & Kernikterus & \\
\hline 1 von 1.000 .000 & Tod & \\
\end{tabular}

Wenn sich ein solches Syndrom abzeichnet, braucht die Frau sehr früh Informationen darüber, was bei den ersten Anzeichen eines Milchstaus zu tun ist, um eine rasche Entwicklung zur Mastitis zu vermeiden. Eine ausführliche Stillanamnese hilft dabei, mögliche Dispositionen zu erkennen.

Daher lautet der Rat, nicht nur zu fragen: „Wie lange haben Sie gestillt?“, sondern auch nach Schmerzen und Beschwerden während der gesamten Stillzeit zu fragen. Tatsächlich berichten sehr viele Frauen, dass sie über die gesamte Stillzeit (also nicht nur in der Anpassungszeit) leichte Schmerzen an den Brustwarzen oder häufig einen Milchstau hatten. Vermutlich gibt es den Vasospasmus bzw. das Raynauld-Syndrom in der Praxis häufiger, als bislang angenommen, wenn auch selten in dieser ausgeprägten Form. Diese Frauen brauchen möglichst frühzeitig Informationen zum Thema Milchstau bzw. Mastitis. In der Leitlinie sind weder Vasospasmus noch Raynauld-Syndrom als Verursacher einer Mastitis aufgeführt.

\section{Risiko: Hyperbilirubinämie}

$60 \%$ aller reifen, gesund geborenen Neugeborenen werden gelb, allerdings brauchen nur 2-3\% eine Therapie [4]. Bereits beim physiologischen Ikterus ist die ganze Aufmerksamkeit der Hebamme gefragt - weil sich manchmal rasant ein pathologischer Ikterus entwickelt. Ein solches Ereignis ist selten: 1 von 100.000 Kindern entwickelt einen Kernikterus ( $\triangleright$ Tab. 2). Selbst bei deutlich erhühten Werten über $50 \mathrm{mg}$ und ausgeprägter Hyperbilirubinämie lassen sich durch einen frühen Therapiebeginn neurologische Schäden vermeiden. Eine rasche Diagnostik gefährdeter Kinder ist wichtig und wird auch von der Leitlinie gefordert [4].

Die AWMF-Leitlinie zur Hyperbilirubinämie empfiehlt bereits seit 2010 ein Neugeborenen-Screening mittels transcutaner Bilirubinmessung $(\mathrm{TcB})$ und ggf. Bestimmung einer blutigen Messung des Gesamt-Bilirubinwerts - zum Beispiel bei der U2. Diese Werte werden in einer
Übersichtsmessskala eingetragen. Nach der Höhe des Wertes wird in einem Bogen vermerkt, wann und durch wen die nächste Kontrolle erfolgen sollte.

Diesen sehr guten Überleitungsbogen der AWMF-Leitlinie [15] kennen viele Hebammen nicht, weil die wenigsten Krankenhäuser ihn nutzen. Dabei eignet sich der Bogen, der mit der Leitlinie downloadbar ist, auch zur Aufklärung der Eltern für die Betreuung im häuslichen Umfeld. Es ist empfehlenswert, Eltern diesen Bogen mitzugeben, damit die geburtshilfliche Abteilung ihn ausfüllt. Dann liefert er der Hebamme einen Entlassungswert, um den weiteren Verlauf des Kindes sicherer beurteilen zu können.

\section{INFO}

\section{Risikofaktoren für Ikterus [4]}

- Klinische Risikofaktoren sind u.a. Blutgruppenunverträglichkeit, Infektionen, Stoffwechselerkrankungen, unzureichendes Stillmanagement, Geburtsverletzungen

- Ethnische Herkunft: Beurteilung der Hautfarbe ist schwierig, Neugeborene mit einem G6PDMangel/Favismus haben ein erhöhtes Risiko für Icterus praecox oder Icterus prolongatus

- Strukturelle/organisatorische Risiken: diagnostische Lücke, mangelnde Zusammenarbeit und Kommunikation, v.a ab Schnittstelle Krankenhaus, häusliches Wochenbett und Frühentlassungen.

\section{G6PD-Mangel - Favismus}

Der krankhafte Verlauf eines G6PD-Mangels wird auch Favismus genannt. Von diesem angeborenen Enzymmangeldefekt sind vor allem Menschen aus dem Mittelmeerraum, dem Nahen Osten und den arabischen Ländern Nordafrikas betroffen. Da Menschen mit G6PD-Mangel eine erhühte Resistenz gegenüber Malariabefall aufweisen, haben sie einen Selektionsvorteil in betroffenen Gebieten. Sie vererben den Mangel 
rezessiv auf dem X-Chromosom weiter. Das heißt: männliche Neugeborene sind häufiger betroffen. Wenn Menschen mit diesem Enzymmangeldefekt bestimmte Lebensmittel verzehren (Gerichte mit Favabohnen/„Saubohnen“) oder bestimmte Medikamente einnehmen (u.a. ASS), bekommen sie vermeintlich eine schwere Magen-Darm-Infektion, in deren Folge eine lebensbedrohliche Hämolyse einsetzt. Das macht diese Krankheit so gefährlich. Für Betroffene ist es wichtig, zu wissen, dass sie diesen Mangeldefekt haben. Sie meiden diese Lebensmittel oder Stoffe bzw. müssen bei Durchfall nach deren Verzehr sofort eine Klinik aufsuchen [14].

Neugeborene, die an einem G6PD-Mangel leiden, haben ein erhöhtes Risiko für einen Icterus praecox oder einen Icterus prolongatus. In Deutschland wird der G6PD-Mangel bislang nicht bundesweit im Stoffwechselscreening erfasst. Zudem liegt bei einem Icterus praecox das Ergebnis des Screenings auch zu spät vor.

Die AWMF-Leitlinie zur Hyperbilirubinämie empfiehlt bereits seit 2010 ein Neugeborenen-Screening mittels transcutaner Bilirubinmessung (TcB) und ggf. Bestimmung einer blutigen Messung des Gesamt-Bilirubinwerts - zum Beispiel bei der U2. Diese Werte werden in einer Übersichtsmessskala eingetragen. Nach der Höhe des Wertes wird in einem Bogen vermerkt, wann und durch wen die nächste Kontrolle erfolgen sollte.

Diesen sehr guten Überleitungsbogen der AWMF-Leitlinie [15] kennen viele Hebammen nicht, weil die wenigsten Krankenhäuser ihn nutzen. Dabei eignet sich der Bogen, der mit der Leitlinie downloadbar ist, auch zur Aufklärung der Eltern für die Betreuung im häuslichen Umfeld. Es ist empfehlenswert, Eltern diesen Bogen mitzugeben, damit die geburtshilfliche Abteilung ihn ausfüllt. Dann liefert er der Hebamme einen Entlassungswert, um den weiteren Verlauf des Kindes sicherer beurteilen zu können.

\section{Fazit}

Neben medizinischen Risiken wie Blutungen oder Infekten tragen auch strukturelle und organisatorische Mängel in der Praxis immer wieder zu riskanten Verläufen im Wochenbett bei.

Riskante Verläufe erfordern von der Hebamme ein ebenso sicheres Handeln wie in der alltäglichen Routine.

Wenn relevante Risiken definiert sind, lässt sich damit arbeiten und es kann der Blick auf das Gesunde weiterhin im Vordergrund stehen. 
Durch fachliche, strukturelle und organisatorische Vorkehrungen sichert die freiberufliche Hebamme einen reibungslosen Ablauf in der alltäglichen Versorgung und in Notfallsituationen.

Risikomanagement im Wochenbett bedeutet:

- Auffrischen und Erwerb von Fachwissen, u.a. Notfalltraining

- Dokumentation: Aufklärung (auch anhand von Aufklärungsbögen), gründliche Anamnese, RisikoChecklisten, Verlaufsdokumentation

- QM-System Risikomanagement: u.a. Beschreibung riskanter Prozesse

- Aufklärung der Frauen über potenziell lebensbedrohliche Situationen im Wochenbett (gemäß Patientenrechtegesetz, Berufsordnung)

- regionale Vernetzung und gute Kommunikation: Regelmäßiger Austausch mit anderen Hebammen, Fachärzten und Krankenhäusern, z.B. gemeinsame Qualitätszirkel/Fortbildungen

Sich daran zu halten, kostet Zeit und Mühe, lohnt sich aber in jedem Fall, um mögliche Gefahren zu identifizieren und ggf. abzuschaffen.

\section{Literatur}

[1] Reason J: Human error: models and management. BMJ. Band 320, Nr. 7237, 18. März 2000, S. 768-770.

[2] Qualität in der Hebammenarbeit, Landesverband der Hebammen Niedersachsen, Dokument 215

[3] www.awmf.org : Brunstentzündungen in der Stillzeit: Therapie (015-071)

[4] www.awmf.org: Hyperbilirubinämie des Neugeborenen Diagnostik und Therapie (024/007)

[5] www.awmf.org: Peripartale Blutungen, Diagnostik und Therapie $(015 / 063)$
[6] The National Institute for Health and Care Excellence, NICE: Postnatal Care. www.nice.org.uk/guidance/qs37

[7] MBRRACE-UK: Mothers and Babies: Reducing Risk through Autdits and Confidential Enquiries across the UK. www.npeu. ox.ac.uk/mbrrace-uk

[8] Rath, W. Medikamentöse Thromboseprophylaxe in Schwangerschaft und Wochenbett: aktuelle Empfehlungen Die Hebamme; 2012:95ff

[9] Cantwell R, Clutton-Brock T, \& Cooper G et al. Saving Mothers' Lives: Reviewing maternal deaths to make motherhood safer: 2006-2008. The Eighth Report of the Confidential Enquiries into Maternal Deaths in the United Kingdom. BJOG. 2011

[10] Stiefel A. Geist C, Harder U (Hrsg.): Hebammenkunde, Yvonne Bovermann, Dorothea Tegethoff : Stillprobleme. Stuttgart: Hippokrates; 2013: 549

[11] Schatten \& Licht e. V. Krise rund um die Geburt. Initiative peripartale psychische Erkrankungen. www.schatten-und-licht.de

[12] Marcé Gesellschaft für Peripartale Psychische Erkrankungen. www.marce-gesellschaft.de

[13] Salis B Psychische Störungen im Wochenbett. Möglichkeiten der Hebammenkunst. München/Jena: Elvesier Urban \& Fischer: 2007

[14] Petri S. www.favismus.de

[15] AWMF: Hyperbilirubinämie des Neugebeorenen - Diagnostik und Therapie; S 11: Blatt zur Einlage in das gelbe Untersuchungsheft

\section{Autorinnen/Autoren}

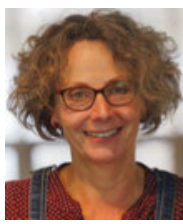

Christiane Borchard ist freiberufliche Hebamme, Familienhebamme und Dipl. Pflegewirtin $(\mathrm{FH})$ in Münster. Sie doziert und referiert $\mathrm{u}$. a. über Notfälle in der Hebammenarbeit

\section{Korrespondenzadresse}

E-Mail: heb.bo@web.de 